\title{
EL CRITERIO DE "VERDAD EFECTIVA" DE NICOLÁS MAQUTAVELO
}

\author{
Ambrosio Velasco Gómez
}

Programa de investigactón ENEP-ACATLAN

\section{Introducción}

El propósito de esta ponencia es elucidar las relaciones entre validez del conocimiento político y eficacia de la acción política en el pensamiento de Nicolás Maquiavelo. Para ello se parte del análisis del criterio de "Verdad efectiva", enunciado por Maquiavelo en El Principe.

El análisis de este criterio se desarrolla en dos apartados. Primeramente, se trata de aclarar el sentido de "Verdad efectiva". En esta primera parte se explicitan algunos presupuestos ontológicos de la teoría maquiaveliana. La segunda parte está dedicada a exponer las implicaciones metodológicas más importantes del criterio de "Verdad efectiva".

A manera de conclusiones, se exponen un conjunto de tesis y argumentos que recapitulan las implicaciones ontológicas y epistemológicas del criterio maquiaveliano de "Verdad efectiva", enfatizando el principio de que la validez del conocimiento y la eficacia de la acción política son una y la misma cosa.

\section{Significado del criterio de "Verdad efectiva"}

La expresión "Verdad efectiva" la utiliza Nicolás Maquiavelo en el capítulo XV de El Príncipe. Sin embargo, el sentido de esta expresión está presente en todas sus obras políticas e históricas. Incluso podríamos afirmar que este criterio no es exclusivo de Maquiavelo sino que pertenece a la cultura del Renacimiento italiano.

La cita donde aparece la expresión "Verdad efectiva" es la siguiente:

Réstame tratar de la conducta y procedimientos que debe seguir un príncipe con sus súbditos y con sus amigos. Sé que muchos han escrito de este asunto y temo que al hacerlo ahora yo, separándome de las opiniones de los otros se me tenga por presuntuoso. Pero mi intento es escribir cosas útiles a quienes las lean y juzgo más conveniente irme derecho a la verdad efectiva de las cosas que como se las imagina; 
porque muchos han visto en su imaginación repúblicas y principados que jamás existieron en la realidad. Tanta es la distancia de cómo se vive y cómo se debería vivir que quien prefiere a lo que se hace lo que debería hacerse, más camina a su ruina que a su preservación y el hombre que quiere portarse en todo como bueno por necesidad fracasa entre tantos que no lo son, necesitando el príncipe que quiere conservarse aprender a poder ser no bueno y a usarlo o no usarlo según la necesidad.1

Para esclarecer el significado contextual del criterio de "Verdad efectiva", conviene privilegiar los conceptos de conducta (o procedimiento, o acción), utilidad y necesidad.

La "conducta" y los "procedimientos" políticos constituyen los referentes fundamentales de la teoría maquiaveliana. Sobre este conocimiento de la acción política se aplica el criterio de "Verdad efectiva".

Maquiavelo opone la connotación de "utilidad" a la meramente axiológica de "deber ser". Con ello se aparta de aquellas perspectivas de estudio que analizan la acción política en términos de la relación lógica entre acciones y principios éticos con pretensión de universalidad (ley natural, ley divina). Esta forma de considerar la política conduce a la construcción de Estados que sólo existen de palabra y en la imaginación. Contrariamente a esta perspectiva axiológica, Maquiavelo insiste en que la validez del conocimiento político implica la eficacia de éste para realizar los fines de la acción.

En consecuencia con lo anterior, puede afirmarse que, para que el conocimiento sobre la acción política sea verdadero, es necesario que se derive en estrategias para la acción política eficaz. En este sentido, la teoría política válida es un conocimiento de y para la acción politica.

La tercera connotación relevante que debemos analizar es la "necesidad". Ésta es una propiedad de las relaciones que subyacen a las circunstancias políticas y que manifiesta su poder causal sobre ellas. La "necesidad" no se observa directamente en las circunstancias, sino que es necesario realizar una interpretación metódica de éstas para descubrirla.

Las circunstancias o situaciones son, en el sentido maquiaveliano, un conjunto de agentes (individuos, familias, grupos, partidos, clases sociales o estados), acciones, medios (instituciones, leyes, ejércitos, dinero), fines (intenciones, intereses) y condiciones geográficas.

1 Maquiavelo, Nicolás, El principe, traducción de Luis Arocena, Madrid, ediciones de la Universidad de Puerto Rico, Revista de Occidente, 1955, Cap. XV, p. 342. En italiano la expresión de "Verdad efectiva" corresponde a "Veritá effectuale". (cfr. Niccoló Machiavelli, Il Principe, Torino, Giulio Einaudi Editore, 1974, Cap. XV, p. 75.) Los subrayados en la cita son míos. 
Las relaciones que estructuran a estos elementos en determinados puntos espacio-temporales constituyen las condiciones.

Las condiciones ejercen un poder causal sobre el resultado de las acciones. Este poder causal es la "necesidad". Así pues, la necesidad es una fuerza causal que determina las consecuencias de las acciones sobre las circunstancias y condiciones a las que las acciones pertenecen.

Para determinadas acciones con determinados medios y fines, las condiciones pueden ser oportunas o inoportunas, según la necesidad haga posible o no la realización de los fines de la acción. Actuar oportunamente es actuar conforme a la necesidad, y si se actúa conforme a la necesidad, se actúa eficazmente.

Las circunstancias, y las condiciones a ellas asociadas, se transforman por efecto de las acciones mismas. El resultado de estas transformaciones puede coincidir o no con las previsiones y los fines de la acción. Si son coincidentes, entonces la acción propositiva del hombre se constituye en fuerza causal del cambio. Por el contrario, si las nuevas circunstancias no han sido previstas por algún agente, entonces es la "fortuna" la causante del cambio de "las cosas de este mundo"."2

Dado que las condiciones afectan causalmente los resultados de la acción, al cambiar las condiciones cambian los resultados de un tipo de procedimiento político. Aś, por ejemplo, en la condición de la antigua Roma era necesario satisfacer prioritariamente los intereses del ejército para que el emperador pudiese conservar el poder. En la época de Maquiavelo este comportamiento llevaría al príncipe a perder el poder $y$ a arruinar al Estado. ${ }^{3} \mathrm{O}$ bien, otro ejemplo, el establecimiento de un régimen monárquico es conveniente en una sociedad donde exista gran desigualdad, pero resulta inadecuado en una sociedad igualitaria."

El devenir de las circunstancias y las condiciones constituye lo que Maquiavelo llama "el tiempo". En el tiempo existen diferentes condiciones, diferentes relaciones causales que a veces operan unas y a veces otras. Debido al cambio de la "condición de los tiempos", cambia también el éxito de las acciones.

2 Maquiavelo, en el Cap. XXV, de El Principe expone con claridad la dialéctica entre fortuna y virtud humana. La virtud humana es la acción previsora y eficaz; ahí donde ésta aumenta, disminuye la fuerza de la fortuna y viceversa: “... la fortuna demuestra su poder cuando no hay virtud ordenada que la resista y con mayor ímpetu donde se sabe que no hay reparo alguno para contrarrestarla." (N. Maquiavelo, op. cit., Cap. XXV, p. 445). También en la Historia de Florencia Maquiavelo mantiene la misma idea: "La adversidad de la fortuna puede vencerse con la prudencia..." (N. Maquiavelo, Historia de Florencia, Madrid, editorial Alfaguara, 1979, Libro III, Cap. 5, pp. 158-159).

3 Cfr. N. Maquiavelo, El principe, Cap. XXV, p. 446.

4 Cfr. N. Maquiavelo, "Discursos sobre la Primera Década de Tito Livio" en sus Obras Politicas, La Habana, Editorial de Ciencias Sociales, 1971, Libro I, Cap. LVI, p. 142. 
...En mi sentir prospera todo el que procede conforme a la condición de los tiempos, y se pierde el que hace lo contrario... De aquí nace, como he dicho, que dos, obrando de distinto modo, uno consiga su propósito y otro no; de aquí también resultan las variaciones del éxito, porque hay tiempos en que las precauciones y la prudencia son buenas y al príncipe que usa de ellas le aprovechan; pero si los tiempos cambian y él no varía de conducta, se arruina.s

Adecuar el comportamiento a "la condición de los tiempos" es requisito suficiente para que la acción sea eficaz. Puede ser que esta conformidad se dé por casualidad o bien que resulte del conocimiento de las condiciones del tiempo. En el primer caso, el éxito resulta de la fortuna; en el segundo, el éxito es resultado de la virtud. ${ }^{\circ}$ Actuar virtuosamente es actuar con base en el conocimiento de las condiciones del tiempo, adecuando los procedimientos a éstas. Este tipo de acciones constituye lo que Maquiavelo denomina "empresas".

Resumiendo lo anterior, podemos decir que el conocimiento político es "efectivamente verdadero" si y sólo si se cumplen las siguientes condiciones:

a) Identificar las condiciones del tiempo, a partir de las circunstancias;

b) Prever los resultados de ciertas acciones en determinadas circunstancias;

c) Diseñar empresas cuyo éxito confirma la validez de las previsiones.

Las connotaciones de utilidad, necesidad y eficacia del criterio de "Verdad efectiva" de Maquiavelo son coincidentes con las connotaciones de criterios epistemológicos propuestos por otros hombres renacentistas de otras disciplinas. Por ejemplo, Leonardo da Vinci, en el capítulo referente a la ciencia en general de su Tratado de la pintura, afirma que todos los efectos que podemos percibir están ligados necesariamente a una causa, y que la identificación de esa causalidad necesaria constituye el propósito fundamental de la ciencia de la naturaleza. También considera que la utilidad de las ciencias determina su jerarquía:

- Maquiavelo, N., El Principe, Cap. XXV, p. 446.

- El éxito por la fortuna es eff́mero, inconstante, porque el agente no sabe cuándo cambian las condiciones del tiempo y por lo tanto no cambia su comportamiento y en consecuencia su fortuna varía. Por el contrario, el éxito por virtud es constante, más duradero, porque conforme cambian los tiempos, se puede, gracias al conocimiento de las nuevas condiciones, adecuar el comportamiento a éstos.

7 " $O$ Oh sorprendente necesidad! obligas por tu ley a que todos los efectos participen de su causa por el camino más corto..." (Leonardo da Vinci, Tratado de la Pintura, Morelia, México, Editorial Balsal, 1978, Cap. II, parágrafo 6, p. 21). Y más 
La ciencia instrumental o mecánica es nobilísima y se eleva por su utilidad, sobre todas las restantes. ${ }^{8}$

Respecto al problema de la producción deliberada de los efectos, Leonardo afirma que nuestros juicios nos engañan cuando "prometen efectos que no pueden tener su causa. en la experiencia. De ahí que, dado un principio, sea necesario que su consecuencia se derive de ella naturalmente y sin obstrucción".?

Estas mismas coincidencias se manifiestan en autores como Copérnico, Galileo y Nicolás de Cusa. Coincidencias que no son casuales sino que resultan de la dependencia de estos autores a un "bloque cultural" del cual son, al mismo tiempo, expresión y forjadores. ${ }^{10}$

\section{Implicaciones metodológicas del criterio de "Verdad efectiva"}

Hemos afirmado en el inciso anterior que, en la perspectiva maquiaveliana, el conocimiento político válido debe ser un conocimiento de "la condición de los tiempos". El problema que queremos estudiar en este inciso es el referente a ¿cómo se puede conocer la "condición de los tiempos"?

El camino seguido por Maquiavelo para conocer verdaderamente los eventos políticos es, según su propio juicio, original:

...he determinado entrar por vía que, no seguida por nadie hasta ahora, me será difícil y trabajosa...

Si la pobreza de mi ingenio, mi escasa experiencia de las cosas presentes y las incompletas noticias de las antiguas, hacen esta tentativa defectuosa y no de grande utilidad, al menos enseñaré el camino a alguno que con más talento, instrucción y juicio, realice lo que ahora intento... ${ }^{11}$

La novedad de su método consiste fundamentalmente en su modali-

adelante afirma: “...así proceden los observadores de los los efectos naturales, y aunque la Naturaleza proceda de la razón y acabe por los efectos, debemos proceder nosotros inversamente, pues procediendo todo, según acabo de decir, de la experiencia, por ella descubrimos la causa." (Ibidem, Cap. II, parágrafo 27, p. 23)

- Da Vinci, Leonardo, op. cit., Cap. II, Parágrafo 27, p. 24.

- Ibidem, cap. II, parágrafo 24, . 24.

10 El problema del condicionamiento cultural del pensamiento maquiaveliano no se detalla en este trabajo. Anteriormente, en otro articulo escrito junto con Lian Karp, hemos explorado estas relaciones de condicionamiento cultural (cfr. Lian Karp y Ambrosio Velasco, "El Príncipe de Maquiavelo", en Cuadernos de Investigación No 1, UNAM, ENEP Acatlán, 1983).

11 Maquiavelo, N., "Discursos sobre la Primera D́écada de Tito Livio" en sus Obras Politicas, prólogo, p. 59. 
dad de articular el conocimiento de las circunstancias presentes con las circunstancias del pasado. El conocimiento de las circunstancias del presente lo obtiene de la experiencia que ha tenido como observador y como funcionario de la República Florentina. El conocimiento de las circunstancias antiguas lo consigue por medio de la lectura de la historia.12

Lo que a Maquiavelo le interesa estudiar en la historia, y observar en la experiencia, son las acciones de determinados agentes (príncipes, ciudadanos, magistrados, clases sociales, partidos, etcétera) ante determinadas circunstancias, asi como las consecuencias que de tales acciones se derivan. El comportamiento o acción incluirá no sólo actos empíricamente manifiestos, sino también medios y propósitos. Estos últimos no se manifiestan empíricamente, sino que deben interpretarse a partir de lo empíricamente manifiesto.

La interpretación del sentido de las acciones constituye un proceso hermenéutico. Este proceso implica la reconstrucción (parcial) del sentido común, de la concepción del mundo de los agentes cuyo comportamiento se estudia.

El intento de rescatar "el sentir" de los agentes se puede encontrar en todas las obras fundamentales de Maquiavelo, pero sobre todo en la Historia de Florencia. Es sumamente interesante analizar los discursos que pone en boca de algunos personajes de su Historia. Por ejemplo, en el Libro tercero, Luis Guicciardini, Gonfalonero de la Señoría, intenta convencer a la plebe, al pueblo infimo, de que desista de sus actos vandálicos en contra de los poderosos y de los nobles:

¿Qué otra cosa sino esclavitud vais a sacar vosotros de vuestras propias disenciones, y qué otra cosa sino pobreza vais a sacar de los bienes que nos habéis robado o que continúan robándose? Porque son precisamente ésos los bienes que, gracias a vuestro trabajo, alimentan a toda la ciudad; y si nos despojáis de ellos, no podrán alimentarla, mientras, por otra parte, los que se han apoderado de ellos, por tratarse de cosa mal adquirida, no sabrán hacerlos continuar produciendo; de donde se seguirán el hambre y la miseria para la ciudad.13

Este discurso contrasta con otro que Maquiavelo pone en labios de uno de los miembros "más decidido y experimentado de la plebe", tendiente a animar a los pobres del pueblo a continuar con los robos y la violencia, destruyendo los argumentos expuestos en el discurso del presidente de la Señoría.

12 También en El Principe Maquiavelo insiste en que las fuentes de su conocimiento son: "...larga experiencia de los asuntos públicos de estos tiempos y no in. terrumpida lectura de los antiguos."

13 Maquiavelo, N., Historia de Florencia, Libro III, cap. XI, p. 170. 
...si observáis el modo de proceder de los hombres, veréis que todos aquellos que han alcanzado grandes riquezas y gran poder, los han alcanzado, o mediante el engaño o mediante la fuerza; y luego, para encubrir lo ilícito de esa adquisición, tratan de justificar con el falso nombre de ganancias lo que han robado con engaños y con violencias. Por el contrario, los que por poca vista, o por demasiada estupidez, dejan de emplear estos sistemas, viven siempre sumidos en la esclavitud y en la pobreza, ya que los siervos fieles son siempre siervos y los hombres buenos son siempre pobres. Los únicos que se liberan de la esclavitud son los infieles y los audaces, y los únicos que se libran de la pobreza son los ladrones y los tramposos. Dios y la naturaleza han puesto todas las fortunas de los hombres en medio de ellos mismos, y éstas quedan más al alcance del robo que del trabajo, y más al alcance de las malas que de las buenas artes. De aquí nace el que los hombres se coman los unos a los otros y que el más débil se lleve siempre la peor parte. Se debe pues emplear la fuerza siempre que se presente la ocasión; y esa ocasión no nos la puede ofrecer mejor fortuna ... de este modo, o quedaremos enteramente dueños de la ciudad o conseguiremos una parte tán importante de ella que no solamente nos perdonarán nuestras faltas pasadas, sino que tendremos fuerza para poder amenazarlos con nuevos daños..." 14

Al ubicar a los agentes políticos en el sentido común que les es propio, Maquiavelo puede comprender las acciones que se desarrollan, pero no explicarlas. La explicación de la acción política, desde el punto de vista maquiaveliano, consiste en dar la razón por la cual se realiza o no el propósito de la acción.

Para decir por qué ciertas acciones triunfan o fracasan respecto a la realización de sus fines, Maquiavelo recurre a ciertas reglas de acción política. Así, al preguntarse por qué Luis XII de Francia perdió la región de Lombardía, que antes había conquistado, la respuesta es "... por no cumplir ninguna de las reglas observadas por cuantos adquirieron provincias con deseos de conservarlas en su poder..." ${ }^{15}$ En ésta y en la mayoria de las explicaciones que expone, pueden encontrarse tres tipos de condiciones fundamentales:

1) Comprensión de la acción en términos de su intencionalidad.

2) Identificación de las circunstancias en donde la acción ocurre.

3) Postulación de un conjunto de reglas de acción que establezcan relaciones de necesidad (o al menos altamente probables), entre comportamientos, circunstancias y consecuencias. 
La primera condición es obvia, ya que es imposible explicar por qué se logra o no el fin propuesto, si no se ha identificado tal fin. El segundo requisito constituye una tipificación de las circunstancias para delimitar la clase de reglas que pueden aplicarse. El tercero equivale a la exigencia de que se disponga de leyes universales (o al menos probabilísticas), que establezcan una relación de causalidad entre acciones, circunstancias y consecuencias.

Las reglas de la acción política son la formulación pragmática de las leyes que rigen causalmente en la acción política; el conocimiento de estas reglas implica el conocimiento de la fuerza causal de "la condición de los tiempos". Consecuentemente, si se analiza la fundamentación y el uso de las reglas de acción, se pueden discernir los procedimientos metodológicos para el conocimiento de "la condición de los tiempos". Analicemos el siguiente texto donde se enuncia una regla de acción:

...La experiencia ha demostrado que Francia fue causante de que creciera el poder en Italia de la Santa Sede y de España, y de este crecimiento procedió su ruina. De aquí se deduce una regla general que nunca o rara vez falla: la de que quien ayuda a otro a engrandecerse trabaja en daño propio, porque el auxilio se lo presta o con esfuerzo $o$ con habilidad, y ambos medios infunden sospechas a quien llega a ser poderoso. ${ }^{16}$

Obsérvese que la regla propuesta está fundamentada en dos tipos de argumentación. Por una parte, se ofrece un caso histórico (el de Francia) como evidencia empírica de la validez de la regla. Por otra, se elabora un argumento de carácter teórico en el que la regla en cuestión se presenta como consecuencia lógica de otras reglas previamente aceptadas. Esta doble manera de argumentar la validez de las reglas es muy común en la obra de Maquiavelo. ${ }^{17}$ En particular, me interesa analizar la argumentación empírica.

La argumentación empírica, que Maquiavelo propone en éste y en muchos otros casos, es muy especial: expone tan sólo unos cuantos casos concretos donde las acciones, las circunstancias y las consecuencias son - supuestamente las mismas (o al menos muy semejantes), y a partir de ellos infiere la regla general. La aplicación de ésta a nuevos casos requiere establecer una analogía entre el nuevo caso y aquellos acontecimientos que sirvieron de fundamento a la regla.

16 tbidem, p. 219.

17 Sobre todo en El Principe, Los Discursos... y El arte de la Guerra. En la Historia de Florencia, las reglas no se exponen y argumentan, sino fundamentalmente se utilizan en explicaciones de acontecimientos. 
En todo caso, sea para fundamentar una regla, o bien, sea para aplicarla, es necesario establecer una relación de analogía entre sucesos de diferente tiempo. Gracias a la relación de analogía, el suceso político bien conocido se convierte en "modelo" del suceso menos conocido y que se quiere explicar. ${ }^{18}$ Maquiavelo utiliza a veces acontecimientos del pasado, como modelo para la explicación de acontecimientos del presente, ${ }^{19}$ pero también en varias ocasiones recurre a acontecimientos del presente como modelo para explicar sucesos del pasado. ${ }^{20}$

Estas relaciones de analogia y modelaje que Maquiavelo utiliza, lo comprometen con una tesis ontológica muy importante, la de que existen poderes causales que perduran en el tiempo. ${ }^{21}$ En los propios términos maquiavelianos, esta tesis se expresa así:

...a la mayoría de los lectores (de la historia) les agrada enterarse de la variedad de sucesos que narra, sin parar mientes en imitar las grandes acciones, por juzgar la imitación no sólo difícil sino imposible; como si el cielo, el sol, los elementos, los hombres, no tuvieran hoy el mismo orden, movimiento y poder que en la antigüedad. ${ }^{22}$

$18 \mathrm{El}$ concepto de modelo que aqui se utiliza no es el que se entiende en el ámbito de la lógica, sino que nos apegamos más al sentido que se le da en la concepción realista de la ciencia. Desde este enfoque, un modelo es "... un análogo hipotético de un mecanismo real aún desconocido. El modelo es en sí mismo modelado a partir de cosas y procesos que ya conocemos... Se trata de una instancia creativa en la construcción de la teoria, en la cual suponemos analogías entre el mecanismo real que nos proponemos conocer y otro mecanismo ya comprendido por una teoria." (R. Harré, The Principles of Scientific Thinking, Chicago Press, p. 35.)

19 Para mencionar el mismo ejemplo de Luis XII de Francia, podemos ver en este caso que las reglas que no observó fueron justamente aquellas que los romanos siguieron en la expansión de su territorio. (Cfr. Maquiavelo, El Principe, cap. III, p. 214.)

20 De este tipo de modelajes, puede citarse la explicación de Maquiavelo sobre la duración del imperio de Alejandro Magno, aún después de muerto éstè. Para explicar este hecho, Maquiavelo recurre a las características de dos tipos de gobiernos de su tiempo: El Gran Turo y el Rey de Francia, explicitando las reglas que rigen para la conquista y conservación de cada uno de estos estados "Quien examinó ambas formas de gobierno encontró mayores dificultades para conquistar el Estado turco; pero una vez vencido, gran facilidad para conservarlo." Después de establecidas las reglas, afirma la analogla entre el Estado del turco y el reino de Dario, conquistado por Alejandro: "El gobierno de Darfo, si bien se considera, resulta de naturaleza semejante al reino del turco", y concluye después con la aplicación de las reglas sobre el Estado turco, al caso del Imperio de Alejandro Magno (cfr. N. Maquiavelo, El Príncipe, cap. IV, pp. 229-232).

21 La afirmación de que existen estructuras subyacentes a los fenómenos, y que tales estructuras tienen poderes causales sobre éstos, es una tesis que desde la filosoffa de la ciencia contemporánea podemos caracterizar de "realista". En particular, el "Realismo Trascendental" de R. Bhaskar en su libro A Realistic of Science, sustenta la tesis de que la empresa científica tiene como propósito descubrir los mecanismos causales de los eventos, mecanismos que existen independientemente de que sean conocidos.

22 Maquiavelo, N., "Discursos sobre la Primera Decada de Tito Livio" en op. cit., prólogo, p. 60. 
Este procedimiento de analogías y modelos se lleva a cabo a través de un proceso dialógico que Maquiavelo expresa de forma muy emotiva en una carta a su amigo Vettori, en la que le comunica que ha terminado una "obrita, De Principatibus":

... anochecido, vuelvo a mi casa y entro en el escritorio; y en el umbral me despojo de las vestiduras cotidianas cubiertas de fango y lodo y me visto reales y curiales ropas; y decentemente vestido penetro en las viejas cortes de los antiguos en las que recibido amablemente por ellos, me nutro del manjar, que es mi manjar y para el cual nací. Allí no me avergüenzo de platicar con ellos y de pedirles justificación de sus acciones ni ellos tan humanos dejan de contestarme y se me pasan como un soplo cuatro horas, en las cuales olvido todos mis afanes, no temo la pobreza ni la muerte me causa pavor; tanto me identifico con ellos. $Y$ como Dante dice que no hay ciencia en donde no se ha retenido lo que se oyó, he anotado aquello que en mis pláticas con ellos vino a enriquecer mis conocimientos... ${ }^{23}$

Este diálogo involucra un procedimiento eminentemente hermenéuti$\mathrm{co}^{24}$ en el cual se cuestionan los textos antiguos y las experiencias ahí manifiestas, desde los intereses y preocupaciones del presente. Las respuestas a estas preguntas transforman los conocimientos sobre el presente (modelaje: antigüedad-presente), los cuales a su vez permiten realizar nuevas in'terpretaciones del pasado (modelaje: presente-antigüedad). Este proceso tiene un carácter no terminal, y conforme se desarrolla aumenta el conocimiento de las reglas de acción política $\mathrm{y}$, con ello, el conocimiento de la condición de los tiempos, del orden necesario de "las cosas de este mundo".

Gracias al conocimiento de la condición que opera en un determinado tiempo, es posible no sólo explicar los acontecimientos políticos, sino también preverlos.

La previsión tiene una estructura muy semejante a la explicación: conocidas las circunstancias, las opciones de acción y las reglas que vincula a cada alternativa con sus consecuencias, es posible inferir cuál será el resultado que ha de obtenerse si el agente sigue uno $\mathbf{u}$ otro procedimiento. Con base en esta inferencia, el agente puede elegir la alternativa de acción cuyo resultado se aproxime más al fin propuesto.

23 Carta de N. Maquiavelo a Vettori del 10 de diciembre de 1513, reproducida por P. Villori en su Maquiavelo, su vida y su tiempo, España, Editorial Grijalbo, 1975, cap. XXIII, pp. 367-369.

24 En nuestros días, el carácter dialógico de la comprensión es una de las tesis fundamentales de la "hermenéutica filosófica de H. G. Gadamer": ". . .la comprensión es siempre una fusión del horizonte del presente con el del pasado... lo viejo y lo nuevo se desarrollan juntos una y otra vez..." (Hans George Gadamer, Philosophical Hermeneutics, edited by D. E. Linge, University of California, 1977, p. XIX). 
La previsión difiere de la predicción, en cuanto en esta última la acción humana no interviene en la realización del hecho que se predice, mientras que en la previsión la acción humana es determinante sobre la ocurrencia de aquella que se prevé.

El éxito de la acción pasada en la previsión constituye una instancia confirmadora de la, o las reglas utilizadas en la previsión y, recíprocamente, este tipo de confirmación implica la eficacia de la acción política.

\section{Conclusiones}

En este último apartado quiero exponer un conjunto de enunciados que, al mismo tiempo que sinteticen los puntos más relevantes de la ontología y metodologia maquiaveliana, sirvan de argumentación a la tesis básica de que la virtud de la acción política y la verdad del conocimiento político se implican recíprocamente.

1. El conocimiento político al que puede aplicarse el criterio de "Verdad efectiva" es aquel que tiene como referente "la condición de los tiempos".

2. "La condición de los tiempos" es el conjunto de relaciones subyacentes que ordenan las circunstancias políticas. Estas relaciones ejercen una fucrza causal sobre los efectos de la acción.

3. Al cambiar el orden de las circunstancias cambia la condición a ellas asociada y, por ende, cambian los resultados de un mismo tipo de acción. En el devenir de las circunstancias es posible que algunas re-

- laciones causales que operaron en el pasado, pero han dejado de operar, recobren posteriormente su vigencia.

4. El conocimiento de las relaciones que constituyen la condición de los tiempos se expresa en enunciados legaliformes, llamados "reglas" - "preceptos". Estas reglas establecen las consecuencias necesarias, o probables, que se siguen de determinado tipo de acción en determinado tipo de circunstancias.

5. Para el descubrimiento de las reglas del comportamiento político, Maquiavelo recurre a procedimientos metodológicos que, en el lenguaje filosófico contemporáneo, implican la integración de elementos propios de las concepciones naturalistas de las ciencias sociales, y de tesis de concepciones hermenéuticas de esas mismas ciencias. Sin pretender establecer un orden secuencial en la estrategia metodológica maquiaveliana, podemos mencionar los siguientes requisitos para el descubrimiento de las reglas de la acción política. 
a) Descripción e identificación de las circunstancias y acciones políticas en un momento histórico determinado.

b) Interpretación de los fines de tales acciones.

c) Descripción de los efectos, observados o previstos, de las acciones.

d) Establecimiento de analogias entre tales acciones, fines o circunstancias, y otras de diferente tiempo, para resolver si a un mismo tipo de acciones, con determinados fines y bajo un mismo tipo de circunstancias, corresponde un mismo efecto. Si tal es el caso, las acciones de ese tipo se proponen como "reglas" para alcanzar ciertos fines en determinadas circunstancias.

6. La validación de las reglas está en función de las explicaciones y sobre todo de las previsiones.

7. Las explicaciones de los acontecimientos políticos tienen la estructura de explicaciones nomológico-deductivas, o al menos la estructura de explicaciones probabilísticas (según que la o las reglas, que operan a manera de leyes en el explanans, admitan o no excepciones). La utilización de las reglas para la explicación de un acontecimiento político requiere del establecimiento de analogías entre ese acontecimiento y otro mejor conocido y ya explicado, que opera como modelo.

8. Las explicaciones amplían el campo de aplicación de la regla y contribuyen secundariamente a su "confirmación". La piedra de toque para "la verificación" de las reglas es la previsión.

9. La estructura de la previsión es muy semejante a la de la predicción, con la salvedad de que en la previsión existe una pluralidad de resultados previstos y la posibilidad de determinar por medio de la acción cuál de esos resultados ha de suceder.

10. El éxito de la acción fundada en la previsión verifica las reglas involucradas en tal previsión, y dado que sólo con reglas válidas la acción política puede ser previsoramente eficaz (y no simplemente afortunadamente eficaz), el incremento de la validez de las reglas $y$ el aumento del poder de la acción propositiva del hombre sobre el curso de la historia (virtud), se implican recíprocamente.

11. De esta manera, bajo el criterio de verdad efectiva, la acción politica virtuosa y el conocimiento político verdadero se funden en un mismo proceso: la conquista de la libertad humana para dirigir el devenir de las cosas de este mundo. 